\title{
Estimation of One Parameter Exponential Family Under A Precautionary Loss Function Based on Record Values
}

\author{
Jinping $\mathrm{li}^{\mathrm{a}}$, Haiping Ren ${ }^{\mathrm{b}}$ \\ ${ }^{\mathrm{a}}$ College of Information Science and Technology, Hainan University, Haikou,570228, China \\ ${ }^{\mathrm{b}}$ School of Software, Jiang Xi University of Science and Technology, Nanchang, 330013, China
}

\begin{abstract}
In this paper, we consider one-parameter exponential family and obtain the Bayes and. empirical Bayes estimators of the unknown parameter based on record values under a precaution asymmetry entropy loss function. The admissibility and inadmissibility of a class of inverse linear estimators of are studied based on upper records.
\end{abstract}

Index Terms: Admissibility, Bayes and empirical Bayes estimators, record values, asymmetry loss function

(C) 2012 Published by MECS Publisher. Selection and/or peer review under responsibility of the Research Association of Modern Education and Computer Science.

\section{Introduction}

A great deal of research has been done on estimating the parameters of these distributions using both classical and Bayesian techniques, and a very good summary of this work can be found in Johnson, Kotz, and Balakrishnan (1994). Record statistics is first studied by Chandler (1952) and is widely used in statistical modelling and inference such as in several real-life problems involving data relating to weather, sports, economics, and life tests.And it has now spread in different directions. There are also some papers on estimation and prediction for parameters of some life distributions based on record values.See for example Balasubramanian and Balakrishnan (1992), Jaheen (2004), and Ahmadi, Doostparast, and Parsian (2005) and references therein.

Let $X_{1}, X_{2}, \cdots$ be a sequence of independent and identically distributed (iid) random variables with cumulative distribution function (cdf) $F(x)$ and probability density function (pdf) $f(x)$. For $n \geq 1$, define

\footnotetext{
* Corresponding author.

E-mail address: 479456011@qq.com, chinarhp@163.com
} 


$$
U(1)=1, U(n+1)=\min \left\{j: j>U(n), X_{j}>X_{U(n)}\right\} .
$$

The sequence $\left\{X_{U(n)}\right\}$ is known as upper record Values.

In this paper, we consider the estimation of a special one-parameter exponential family based on record values. Bayes and empirical Bayes estimators are obtained in Section 2. The estimators obtained are special cases of the more general class of linear estimators of the form $(c T+d)^{-1}$. In Section 3 , the admissibility and inadmissibility of $(c T+d)^{-1}$ are studied. Conclusions and a brief summary of the results are finally provided in Section 4.

Let $X_{1}, X_{2}, \cdots$ be a sequence of iid random variables from the class $\mathrm{C}$ of one-parameter exponential family with cdf

$$
F(x ; \theta)=1-\exp \{-a(\theta) T(x)\}
$$

and pdf

$$
f(x ; \theta)=a(\theta) T^{\prime}(x) \exp \{-a(\theta) T(x)\}, x>0
$$

where $\theta>0$ and $a(\theta), T(x)$ is a real positive function.

The family in $\mathrm{C}$ is well-known in the lifetime experiments which includes several well-known lifetime distributions such as: Exponential, Pareto, Lomax, Burr type XII, Resnick, Weibull (one parameter) among others.

\section{Bayes and Empirical Bayes Estimation}

Suppose we observe n upper record values $X_{U(1)}=x_{1}, \cdots, X_{U(n)}=x_{n}$ from distribution cdf (1) and pdf (2). Then, the joint distribution of $X_{U(1)}, X_{U(2)}, \cdots, X_{U(n)}$ is given (see Arnold et al. (1998)) by

$$
\begin{aligned}
& f(\underline{x} ; \theta)=f\left(x_{n} ; \theta\right) \prod_{i=1}^{n-1} H\left(x_{i} ; \theta\right) \\
& =a^{n}(\theta) \exp \left\{-a(\theta) T\left(x_{n}\right)\right\} \prod_{i=1}^{n} T^{\prime}\left(x_{i}\right)
\end{aligned}
$$

where $0<x_{1}<x_{2}<\cdots<x_{n}$ and the marginal pdf of $X_{U(n)}$ is given by

$$
\begin{aligned}
& f_{n}\left(x_{n} ; \theta\right)=f\left(x_{n} ; \theta\right) \frac{R^{n-1}\left(x_{n} ; \theta\right)}{(n-1) !} \\
& =\frac{a^{n}(\theta)}{(n-1) !} T^{n-1}\left(x_{n}\right) T^{\prime}\left(x_{n}\right) \exp \left\{-a(\theta) T\left(x_{n}\right)\right\}
\end{aligned}
$$


where $\underline{x}=\left(x_{1}, \cdots, x_{n}\right), H(\cdot)$ is the hazard function corresponding to $\operatorname{pdf} f(\cdot)$,

$$
H\left(x_{i} ; \theta\right)=\frac{f\left(x_{i} ; \theta\right)}{1-F\left(x_{i} ; \theta\right)}
$$
and $R(x ; \theta)=-\ln (1-F(x ; \theta))$.

For the later use,we give the following results which can be found their proof in $\operatorname{Ren}(2010)$.

Lemma 2.1 Suppose we observe $\mathrm{n}$ upper record values $X_{U(1)}=x_{1}, X_{U(2)}=x_{2}, \cdots, X_{U(n)}=x_{n}$ from distribution cdf (1.1) and pdf (1.2). Then

1) $X_{U(n)}$ is a sufficient statistics for the first n upper record values.

2) $T\left(X_{U(n)}\right) \sim \Gamma(n, a(\theta))$

Lemma 2.2 Suppose we observe n upper record values $X_{U(1)}=x_{1}, \cdots, X_{U(n)}=x_{n}$ from distribution cdf (1) and pdf(2) and $a(\theta)=\theta$, Then the maximum likelihood estimator (MLE) of $\theta$ is

$$
\hat{\theta}_{M L E}=n / T\left(X_{U(n)}\right)
$$

\section{A. Bayes Estimation}

In the following discussion, we always suppose $X_{1}, X_{2}, \cdots$ be a sequence of iid random variables from the class $\mathrm{C}$ and $a(\theta)=\theta$. And $\mathrm{n}$ upper record values $X_{U(1)}=x_{1}, X_{U(2)}=x_{2}, \cdots, X_{U(n)}=x_{n}$ are also given. we will consider the Bayesian and empirical Bayesian estimation of $\theta$ under a precautionary loss function which proposed by Norstrom(1996), The asymmetric precautionary loss function is

$$
L(\hat{\theta}, \theta)=\frac{(\theta-\hat{\theta})^{2}}{\hat{\theta}}
$$

The loss function approach infinitely near the origin to prevent under estimation, thus giving conservative estimators, especially when low failure rates are being estimated.It is very useful when underestimation may lead to serious consequences.For instance, in the case of estimation of a financial charge or size of an order,underestimation has much more serious conseququences.

The Bayes estimator under precautionary $\operatorname{loss}(5)$ is denoted by $\hat{\theta}_{B}$, and is given by $\hat{\theta}_{B}=\left[E\left(\theta^{2} ! X\right]^{1 / 2}\right.$

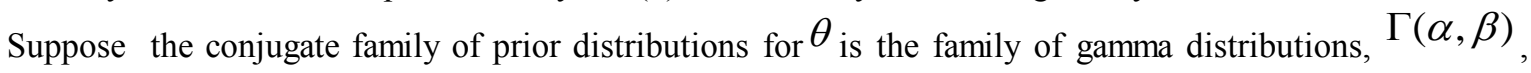
with density

$$
\pi(\theta \mid \alpha, \beta)=\frac{\beta^{\alpha}}{\Gamma(\alpha)} \theta^{\alpha-1} e^{-\beta \theta}, \theta>0
$$

where $\alpha>0$ and $\beta>0$. Note that the limiting case $\alpha, \beta \rightarrow 0$ gives the usual noninformative prior for $\pi(\theta) \propto \theta^{-1}$.Then the posterior distribution of $\theta$ is $\Gamma\left(n+\alpha, \beta+T\left(x_{n}\right)\right)$, and the unique Bayes estimator of $\theta$, say $\hat{\theta}_{B}$, is given by 


$$
\hat{\theta}_{B}=\left[E\left(\theta^{2} \mid \underline{X}\right)\right]^{1 / 2}=\left[\frac{(n+\alpha)(n+\alpha+1)}{\left(\beta+T\left(X_{U(n)}\right)\right)^{2}}\right]^{1 / 2}
$$

That is

$$
\hat{\theta}_{B}=\left[\frac{T\left(X_{U(n)}\right)}{\sqrt{(n+\alpha)(n+\alpha+1)}}+\frac{\beta}{\sqrt{(n+\alpha)(n+\alpha+1)}}\right]^{-1}
$$

which is of the form $\left[c T\left(X_{U(n)}\right)+d\right]^{-1}$

Remark 2.1. For the noninformative prior $\pi(\theta) \propto \theta^{-1}$, the posterior distribution of $\theta$ is $\Gamma\left(n, T\left(x_{n}\right)\right)$ and we obtain the generalized Bayes estimator

$$
\hat{\theta}_{*}=\left[\frac{T\left(X_{U(n)}\right)}{\sqrt{n(n+1)}}\right]^{-1}
$$

B. Empirical Bayes Estimation

Assume that the conjugate family of prior distributions for $\lambda=\theta^{-1}$ is the family of gamma distributions, $\Gamma(\alpha, \beta)$ with parameter $\alpha$ and $\beta$, and prior parameter $\beta$ is unknown. we may use the empirical

Bayes approach to get its estimate. From (3) and (6), we calculate the marginal pdf of $\underline{X}=\left(X_{U(1)}, \cdots, X_{U(n)}\right)$, with density

$$
\begin{aligned}
& m(\underline{x} \mid \beta)=\int_{0}^{\infty} f(\underline{x} \mid \theta) \pi(\theta \mid \beta) d \theta \\
& =\int_{0}^{\infty} \theta^{n} \exp \left\{-\theta T\left(x_{n}\right)\right\} \prod_{i=1}^{n} T\left(x_{i}\right) \frac{\beta^{\alpha}}{\Gamma(\alpha)} \theta^{\alpha-1} e^{-\beta \theta} d \theta=\frac{\beta^{\alpha}}{\Gamma(\alpha)} \frac{\Gamma(n+\alpha)}{\left(\beta+T\left(x_{n}\right)\right)^{n+\alpha}} \prod_{i=1}^{n} T\left(x_{i}\right)
\end{aligned}
$$

Based on $m(\underline{x} \mid \beta)$, we obtain an estimate $\hat{\beta}_{\text {of }} \beta$. The MLE of $\beta$ is $\hat{\beta}=\frac{\alpha}{n} T\left(X_{U(n)}\right)$

Now, by substituting $\hat{\beta}_{\text {for }} \beta$ in the Bayes estimator, we obtain the empirical Bayes estimator as

$$
\hat{\theta}_{E B}=\left[\frac{T\left(X_{U(n)}\right)}{\sqrt{(n+\alpha)(n+\alpha+1)}}+\frac{\frac{\alpha}{n} T\left(X_{U(n)}\right)}{\sqrt{(n+\alpha)(n+\alpha+1)}}\right]^{-1}
$$

That is

$$
\hat{\theta}_{E B}=\left[\frac{n+\alpha}{n \sqrt{(n+\alpha)(n+\alpha+1)}} T\left(X_{U(n)}\right)\right]^{-1}
$$




\section{Admissibility of $(c T+d)^{-1}$}

Note that the estimators obtained in Section 2 are special cases of the more general class of linear estimators of the form $\left[c T\left(X_{U(n)}\right)+d\right]^{-1}$. In this section we always let $c_{*}=\frac{1}{\sqrt{n(n+1)}} c^{*}=\frac{1}{\sqrt{n(n-1)}}, T=T\left(X_{U(n)}\right)$

In the rest of this paper, these estimators are compared on the basis of their risks under the loss (2.3). We also obtain conditions under which linear estimators are admissible in terms of risk.

Theorem 3.1. The estimator $(c T+d)^{-1}$ is admissible, provided $0 \leq c<c_{*}$ and $d>0$.

Proof. From (7), when $\alpha>0$ and $\beta>0$, the coefficient of $T$ is strictly between 0 and $c^{*}$, and the constant $\frac{1}{\sqrt{(n+\alpha)(n+\alpha+1)}} \beta$ is strictly bigger than 0 . This proves that $(c T+d)^{-1}$ is admissible for $0<c<c^{*}$ and $d>0$. When $c=0, d>0$, the estimator $(c T+d)^{-1}$ is admissible since it is the only estimator for which $R(\theta, d)=0$ when $\theta=d$

Theorem 3.2 Let the parameter space be ${ }^{(0,+\infty)}$ and the action space be ${ }^{[0,+\infty)}$ The linear estimator $[c T+d]^{-1}$ is inadmissible under the loss function (2.3) whenever one of the following conditions holds:

(i) $c<0$ or $d<0$;

(ii) $0<c<c^{*}$ and $d=0$;

(iii) $c>c^{*}$ and $d \geq 0$

Proof. To see (i), note that $(c T+d)^{-1}$ takes on negative values with positive probability. Therefore $(c T+d)^{-1}$ is dominated by $\max \left(0,(c T+d)^{-1}\right)$ in this case.

For the case (ii), using lemma 2.1, the risk function of $(c T)^{-1}$ is

$$
\begin{gathered}
R\left(\theta,(c T)^{-1}\right)=E_{X \mid \theta}\left[L\left(\theta,(c T)^{-1}\right)\right] \\
=c \theta^{2} E[T]+\frac{1}{c} E\left[\frac{1}{T}\right]-2 \theta=c \theta^{2} \frac{n}{\theta}+\frac{\theta}{c(n-1)}-2 \theta
\end{gathered}
$$

Then derivative of the risk with respect to $\mathrm{c}$ is :

$$
\frac{\partial}{\partial c} R\left(\theta,(c T)^{-1}\right)=n \theta-\frac{1}{c^{2}} \frac{\theta}{n-1}<0,
$$

where $0<c<c^{*}$

Thus the risk of $(c T)^{-1}$ is minimized at $c=c^{*}$. 
Hence the estimator $(c T)^{-1}$ is dominated by $\left(c^{*} T\right)^{-1}$ in this case.

For case (iii), let us compute the risk function of the linear estimator $(c T+d)^{-1}$.

$$
\begin{aligned}
R\left(\theta,(c T+d)^{-1}\right)-R\left(\theta,\left(c^{*} T+\frac{c^{*}}{c} d\right)\right)^{-1} & \theta^{2} E(c T+d)+E\left(\frac{1}{c T+d}\right)-\theta^{2} E\left(c^{*} T+\frac{c^{*}}{c} d\right) \\
& -E\left(\frac{1}{c^{*} T+\frac{c^{*}}{c} d}\right) \\
= & \theta^{2}\left(1-\frac{c^{*}}{c}\right) E(c T+d)+\left(1-\frac{c}{c^{*}}\right) E\left(\frac{1}{c T+d}\right) \\
= & \left(c-c^{*}\right)\left[\frac{\theta^{2}}{c} E(c T+d)-\frac{1}{c^{*}} E\left(\frac{1}{c T+d}\right)\right] \\
\geq & \left(c-c^{*}\right)\left[\frac{\theta^{2}}{c} E(c T+d)-\frac{1}{c^{*}} E\left(\frac{1}{c T}\right)\right. \\
= & \left(c-c^{*}\right)\left[\frac{\theta^{2}}{c} \frac{c n}{\theta}-\frac{1}{c^{*}} \frac{\theta}{c(n-1)}\right] \\
\geq & \left(c-c^{*}\right)\left[n \theta-\frac{1}{c^{* 2}} \frac{\theta}{(n-1)}\right] \\
= & \left(c-c^{*}\right)\left[n \theta-n(n-1) \frac{\theta}{(n-1)}\right]=0
\end{aligned}
$$

Therefore, $R\left(\theta,(c T+d)^{-1}\right.$ is minimized at $c=c^{*}$. Hence $(c T+d)^{-1}$ is dominated by $\left(c^{*} T+d\right)^{-1}$ in this case.

Remark 3.1 Using Theorem 3.3, the estimators $\hat{\theta}_{M L E}$ andb $\hat{\theta}_{E B}$ are inadmissible. They are dominated by the estimator $\hat{\theta}=\left(c^{*} T\right)^{-1}$.

\section{Conclusions}

Based on record statistics, this paper considers the estimation of the unknown parameter from the one parameter exponential family. The maximum likelihood estimator, Bayes and empirical Bayes estimators are obtained. These estimators all belong to the class of inverse linear estimators of the form $(c T+d)^{-1}$, where 
$T=T\left(X_{U(n)}\right)$.So, the admissibility and inadmissibility of $(c T+d)^{-1}$ are studied. As a result, the empirical Bayes estimator and the maximum likelihood estimator are inadmissible.

\section{References}

[1] H. Chandler, K. N. The distribution and frequency of record values. Journal of the Royal Statistical Society. Series B, Vol.14, pp.220-228,1952

[2] Arnold, B. C., Balakrishnan, N., Nagaraja, H. N.Records. New York: John Wiley \& Sons, 1998

[3] Jaheen, Z. F. Empirical Bayes analysis of record statistics based on LINEX and quadratic loss functions. Computers and Mathematics with Applications, Vol.47, pp.947-954,2004

[4] Jaheen Z F .A Bayesian analysis of record statistics from the Gompertz model. Appl Math Comput., Vol.145,pp.307-320,2003

[5] Ahmadi J,Doostparast M. Bayesian estimation and prediction for some life distributions based on record Values. Stat Pap. Vol.47,pp.373-392,2006

[6] A. Asgharzadeh.On Bayesian estimation from exponential distribution based on records.The Korean Statistical Society,Vol.38,no.2,pp.125-130,2009

[7] Ren H.P, Xiao X.Y. Estimation of one parameter exponential family based on records, Proceeding of the 2010 International Conference on Application of Mathematics and Physics, Vol 2,242-247,2010 